\title{
Resistência à mupirocina entre isolados de Staphylococcus aureus de profissionais de enfermagem*
}

\author{
Mupirocin-resistant Staphylococcus aureus in samples belonging to professional nurses
}

Resistencia al mupirocin en cepas aisladas de Staphylococcus aureus de profesionales de enfermería

\author{
Josely Pinto de Moura ${ }^{1}$, Elucir Gir ${ }^{2}$, Juliana de Oliveira Rosa ${ }^{3}$, Fernando \\ Belíssimo-Rodrigues ${ }^{4}$, Elaine Drehmer de Almeida Cruz ${ }^{5}$, Ana Claudia Alves de \\ Oliveira $^{6}$, Fabiana Cristina Pimenta ${ }^{7}$
}

\begin{abstract}
RESUMO
Objetivos: Avaliar a colonização por Staphylococcus aureus sensíveis e resistentes à meticilina na saliva de profissionais de enfermagem e o perfil de resistência à mupirocina. Métodos: Foram coletadas três amostras da saliva de 356 (94,2\%) profissionais participantes da pesquisa, totalizando 1.068 amostras processadas. Todos os aspectos éticos foram contemplados. A análise microbiológica compreendeu a identificação fenotípica, segundo normas do Clinical and Laboratory Standards Institute. Resultados: Foram obtidos 73 indivíduos colonizados por Staphylococcus aureus na primeira coleta, 48 na segunda e 37 na terceira, somando 158 isolados de Staphylococcus aureus. Desses, 24 (15,2\%) eram resistentes à oxacilina e 30 resistentes à mupirocina $(18,9 \%)$. A resistência à mupirocina foi observada de forma pronunciada nos Staphylococcus aureus metacilina resistente (MRSA); dos 24 MRSA, 17 (70,8\%) apresentaram-se resistentes a este antimicrobiano. Conclusão: Os resultados indicaram que a resistência à mupirocina é um importante problema, pois esse antibiótico é um excelente aliado nas medidas de controle das infecções de serviços de saúde. Descritores: Staphylococcus aureus; Resistência a múltiplas drogas; Mupirocina; Equipe de enfermagem
\end{abstract}

\begin{abstract}
Objectives: To assess colonization by Staphylococcus aureus sensitive and resistant to methicillin found in the saliva of nurses and, to recognize the mupirocin profile of resistance. Methods: It was collected three saliva samples of 356 (94.2\%) professional research participants, totaling 1068 samples processed. All ethical aspects were covered. Microbiological analysis included the phenotypic identification, according to the standards of the Clinical and Laboratory Standards Institute. Results: In the first collection were obtained 73 individuals colonized by Staphylococcus aureus, 48 in the second and 37 in the third, totaling 158 samples of Staphylococcus aureus. Of these, 24 $(15.2 \%)$ were resistant to oxacillin and 30 resistant to mupirocin $(18.9 \%)$. Mupirocin resistance was observed sharply in the Staphylococcus aureus resistant to methicillin (MRSA); of 24 MRSA, 17 (70.8\%) were resistant to this antibiotic. Conclusion: The results indicated that mupirocin resistance is a major problem since this antibiotic is an excellent ally in the measures taken to control infection in health services.
\end{abstract} Keywords: Staphylococcus aureus; Drug resistance, multiple; Mupirocin ; Nursing team

\section{RESUMEN}

Objetivos: Evaluar la colonización por Staphylococcus aureus sensibles y resistentes al meticilín en la saliva de profesionales de enfermería y, reconocer el perfil de resistencia a la mupirocina. Métodos: Fueron recolectadas tres muestras de saliva de 356 (94,2\%) profesionales participantes de la investigación, totalizando 1.068 muestras procesadas. Todos los aspectos éticos fueron contemplados. El análisis microbiológico comprendió la identificación fenotípica, según normas del Clinical and Laboratory Standards Institute. Resultados: Fueron obtenidos 73 individuos colonizados por Staphylococcus aureus en la primera recolección, 48 en la segunda y 37 en la tercera, sumando 158 muestras aisladas de Staphylococcus aureus. De esas, 24 (15,2\%) eran resistentes a la oxacilina y 30 resistentes a al mupirocina (18,9\%). La resistencia al mupirocina fue observada de forma pronunciada en los Staphylococcus aureus resistentes a la metacilina (MRSA); de los 24 MRSA, $17(70,8 \%)$ se presentaron resistentes a este antimicrobiano. Conclusión: Los resultados indicaron que la resistencia a la mupirocina es un importante problema, ya que ese antibiótico es un excelente aliado en las medidas de control de las infecciones de servicios de salud. Descriptores: Staphylococcus aureus; Resistencia a múltiples medicamentos; Mupirocina; Grupo de enfermería

\footnotetext{
* Estudo realizado, no periodo de janeiro de 2007 a fevereiro de 2008, nas UTI, clínica médica, clínica cirrirgica e gineco-obstétrica de um hospital universitário de grande porte do interior paulista, (Brasil).

${ }^{1}$ Doutoranda do Programa Interunidades de Doutoramento em Enfermagem da de Ribeirão Preto da Universidade de São Paulo - USP, Ribeirão Preto (SP), Brasil.

${ }^{2}$ Professora Titular da Escola de Enfermagem de Ribeirão Preto da Universidade de São Paulo - USP, Ribeirão Preto (SP), Brasil.

3 Biomédica. Pós-graduanda (Mestrado) em Medicina Tropical (IPTSP/UFG). Instituto de Patologia Tropical e Saúde Pública (IPTSP-UFG). Goiânia, GO, Brasil

${ }^{4}$ Médico Infectologista, Mestre em Clínica Médica, Vice-presidente da Comissão de Controle de Infecção Hospitalar do Hospital das Clínicas da FMRP-USP,

Professor da Faculdade de Medicina do Centro Universitário Barão de Mauá.

${ }^{5}$ Professora Adjunto do Departamento de Enfermagem - UFPR

${ }^{6}$ Doutora. Professora do Instituto de Patologia Tropical e Saúde Pública - UFG

7 Pós-graduanda (Mestrado) Universidade Federal de Goiás.Instituto de Patologia Tropical e Saúde Pública - UFG.
}

Autor Correspondente: Josely Pinto de Moura

R. Prs. Antonio Carlos, 40 - Centro - Passos (MG), Brasil

CEP. 37900-092 E-mail: jpmfonseca@uol.com.br

Artigo recebido em 14/02/2009 e aprovado em 24/11/2009 


\section{INTRODUÇÃO}

Apesar de quase meio século de concentração de esforços, infecções associadas à assistência em saúde permanecem ainda como a principal causa de morbidade e mortalidade e incremento de custos. $\mathrm{O}$ tratamento destas infecções vem se tornando mais complexo em virtude do crescimento alarmante da resistência aos antibióticos.

A incidência de infecções causadas por Staphylococcus aureus meticilina resistente (MRSA) aumentou, inexoravelmente, ao longo da última década, sendo muito mais letais se comparadas às infecções estafilocócicas por germes suscetíveis à meticilina ${ }^{(1)}$.

As estimativas apontam número superior a 94.000 casos de MRSA invasivo na população dos Estados Unidos da América, dos quais 18\% faleceram durante a internação inicial. Aproximadamente, $75 \%$ dos casos eram bacteremias não complicadas; outros incluíam empiema, endocardite e osteomielite. A maioria das infecções invasivas por MRSA, aproximadamente, $86 \%$ ocorreram em indivíduos com exposição a ambientes hospitalares ou de assistência à saúde, e cerca de $14 \%$ ocorreram em indivíduos sem hospitalizações recentes ou qualquer outro fator de risco para MRSA. De acordo com os Centers for Diseases and Control (CDC), o MRSA representou mais de 50\% de infecções por Staphylococcus aureus adquiridas no hospital e 63\% destas infecções foram tranmitidas em Unidade de Terapia Intensiva (UTI) nos Estados Unidos da América ${ }^{(2)}$.

O Staphylococcus aureus caracteriza-se como colonizante de pele e mucosa; quando resistente à meticilina, a descolonização das mucosas e pele deve ser considerada. Mupirocina, antimicrobiano de uso tópico, é recomendado para descolonização da mucosa nasal e lesões cutâneas de pacientes ou profissionais da saúde ${ }^{(3-4)}$. Esta descolonização visa a limitar a disseminação desse agente nos serviços de saúde e, assim, reduzir o grande impacto clínico produzido por ele nas infecções hospitalares, notadamente, aquelas relacionadas a procedimentos cirúrgicos e cateteres vasculares ${ }^{(4)}$.

Estudos avaliaram a vigilância ativa por meio da cultura em swab nasal e/ou de orofaringe e demonstraram que esta medida contribui para redução das taxas de infecção ${ }^{(5-6)}$.

O uso de mupirocina no pré-operatório de 96 pacientes submetidos à esofagectomia radical eletiva com toracotomia e laparotomia demonstrou uma significativa redução no índice de infecção hospitalar entre pacientes que eram portadores de Staphylococcus aureus ${ }^{(7)}$.

$\mathrm{Na}$ Alemanha, a eficácia de um programa que preconizava o isolamento e a terapia de descolonização de portadores de MRSA para a redução das infecções foi comprovada. Resultado semelhante foi também observado em UTI de hospitais canadenses ${ }^{(8-9)}$.

No Brasil, um estudo realizado em uma hemodiálise demonstrou uma redução significativa da bacteremia associada à utilização de cateter venonoso central quando impregnado com mupirocina em comparação ao grupo controle. A bacteremia por Staphylococcus aureus foi identificada em 17 pacientes (dois no grupo de mupirocina [0,71 episódios por 1.000 pacientes-dia] e 15 no grupo de controle [8,92 por 1.000 paciente-dias], $\mathrm{P}<0,001)$. A relação de risco de bacteremia por Staphylococcus aureus 7,2 (95\% intervalo de confiança, 1,6 a 31,6) vezes foi maior em pacientes que não utilizaram mupirocina na saída do cateter ${ }^{(10)}$.

A eficácia de um programa de prevenção e controle de infecção em UTI também no Brasil pós-intervenção de descolonização e vigilância de controle, bem como redução da mortalidade foi demonstrada com índices de redução significativos ${ }^{(11)}$.

A resistência a este antibiótico tem sido descrita em hospitais que fazem uso regular dessa prática ${ }^{(5,12)}$. Já se demonstrou que a resistência é facilmente transferível entre Staphylococcus aureus e exibe relação com o uso prolongado da mupirocina em locais onde o MRSA é endêmico ${ }^{(12)}$.

Um aumento da concentração inibitória mínima de $0,25 \mathrm{mcg} / \mathrm{ml}$ foi descrito antes do tratamento para 8 $\mathrm{mcg} / \mathrm{ml}$ após o tratamento ${ }^{(13)}$. Análise genética do mecanismo de resistência de mupirocina em isolados de Staphylococcus aureus mostrou a presença do gene mup A, detectado de um plasmídeo $38 \mathrm{~kb}^{(8,14)}$.

No Kuwait, pesquisadores descreveram um clone com alta resistência à mupirocina, identificado pela técnica de eletroforese em campo pulsado, em isolados de uma unidade de queimados e disseminado entre cinco outros hospitais ${ }^{(14)}$.

Um contundente documento, sobre o tema, da Organização Mundial de Saúde enfoca que a resistência às drogas está minando a possibilidade do tratamento de muitas doenças infecciosas. Apenas nos Estados Unidos da Amérca, aproximadamente, 14.000 indivíduos são infectados morrem a cada ano, em consequência dos micróbios multirresistentes dquiridos nos hospitais ${ }^{(14)}$.

A coleta de swab nasal e dos dedos de 26 enfermeiros, antes e após as atividades consideradas sujas, mostrou colonização por MRSA de 50\%, evidenciando a colonização relacionada à assistência ${ }^{(15)}$.

Para que a estratégia da vigilância ativa da MRSA e a de colonização dos portadores continue a ser efetiva, é fundamental que se avalie o nível de resistência à mupirocina encontrado nos serviços de saúde.

Este diagnóstico poderá ser realizado em nível local em razão da diversidade do perfil de resistência à mupirocina encontrado na literatura, bem como da heterogeneidade das medidas de controle de MRSA adotadas nas instituições.

Mas o grande problema em questão é a resistência a este antimicrobiano identificada em algumas instituições. Necessitamos do diagnóstico institucional antes da definição do protocolo de controle de MRSA para 
garantirmos a adequação e eficácia da medida.

\section{OBJETIVOS}

Avaliar a colonização por Stapbylococcus aureus sensíveis e resistentes à meticilina na saliva dos profissionais de enfermagem de um hospital de nível terciário.

Avaliar o perfil de resistência à mupirocina encontrado na instituição e analisar a possibilidade de adoção da medida de descolonização de MRSA com este antimicrobiano.

\section{MÉTODOS}

Estudo epidemiológico transversal realizado, no período de janeiro de 2007 a fevereiro de 2008, nas UTI, clínica médica, clínica cirúrgica e gineco-obstétrica de um hospital universitário de grande porte do interior paulista, (Brasil). Optamos por desenvolver a pesquisa nesses locais por atenderem clientes graves, com internação prolongada utilizarem procedimentos invasivos, entre outros agravos à saúde.

Após a aprovação pelo Comitê de Ética em Pesquisa com seres humanos (Processo n ${ }^{\circ}$ 2005/58463-0), iniciamos a coleta de dados. Primeiro convidamos os profissionais da equipe de enfermagem lotados nessas unidades a participar do estudo. Solicitamos que consentissem formalmente em participar, assinando o Termo de Consentimento Livre e Esclarecido. Posteriormente, foram coletadas três amostras de saliva dos profissionais da enfermagem.

A coleta foi realizada nos três turnos de trabalho, com intervalo de dois meses. O estudo contou com 356 participantes, sendo eles: enfermeiros, técnicos e auxiliares de enfermagem.

Estes profissionais foram selecionados por serem considerados os responsáveis diretos pelo cuidado aos clientes; portanto, por manterem contato prolongado com a clientela dessas unidades, o que os tornam potenciais veiculadores de micro-organismos e disseminadores de infecção cruzada.

O espécime clínico foi coletado diretamente em tubo seco, estéril e com tampa. No laboratório, a saliva foi homogeneizada e semeada pela técnica da gota em placas de Petri, contendo meio de cultura seletivo, ágar manitol salgado. As colônias típicas de Staphylococcus aureus foram submetidas aos testes: coloração de Gram, coagulase, catalase, fermentação do manitol, DNase e lecitinase para a diferenciação e identificação de gênero e espécie. Os testes de suscetibilidade aos antimicrobianos foram realizados pelo método de difusão em disco, conforme as recomendações do Clinical and Laboratory Standards Institute $e^{(15)}$.

A análise dos dados foi realizada por meio da estatística descritiva, utilizando o programa Excel como banco de dados dos resultados.

\section{RESULTADOS}

Participaram do estudo 356 profissionais da equipe de enfermagem, totalizando 1.068 culturas processadas. Em 910 (85,2\%), não houve identificação de Staphylococcus aureus na saliva, e em $158(14,8 \%)$ culturas observou-se crescimento bacteriano deste micro-organismo, sendo 73 na primeira coleta, 48 na segunda e 37 na terceira. Dos 158 isolados, $24(15,2 \%)$ foram caracterizados como MRSA e 134 (84,8\%) como Staphylococcus aureus sensíveis à meticilina (MSSA) (Tabela 1).

No presente estudo, observamos que, entre um total de 158 amostras 30(18,98\%) apresentaram resistência à mupirocina, dentre estes 17 MRSA apresentaram maior resistência à mupirocina $(70,8 \%)$ do que os 13 MSSA $(9,7 \%)$ (Tabelas 2 e 3).

Tabela 2 - Perfil de resistência à mupirocina dos Staphylococcus aureus resistentes à meticilina (MRSA), segundo as três coletas. Ribeirão Preto, 2007-8

\begin{tabular}{|c|c|c|c|c|c|c|c|c|}
\hline \multirow{3}{*}{ Mupirocina } & \multicolumn{6}{|c|}{ MRSA } & \multirow{2}{*}{\multicolumn{2}{|c|}{ Total }} \\
\hline & \multicolumn{2}{|c|}{$1^{a}$} & \multicolumn{2}{|c|}{$2^{a}$} & \multicolumn{2}{|c|}{$3^{a}$} & & \\
\hline & n. ${ }^{\circ}$ & $\%$ & n. ${ }^{\circ}$ & $\%$ & n. ${ }^{\circ}$ & $\%$ & n. ${ }^{\circ}$ & $\%$ \\
\hline Sensível & 5 & 27,8 & 1 & 33,3 & 1 & 33,3 & 7 & 29,2 \\
\hline Resistente & 13 & 72,2 & 2 & 66,7 & 2 & 66,7 & 17 & 70,8 \\
\hline Total & 18 & 100,0 & 3 & 100,0 & 3 & 100,0 & 24 & 100,0 \\
\hline
\end{tabular}

Tabela 3 - Perfil de resistência à mupirocina dos Staphylococcus aureus sensíveis à meticilina (MSSA), segundo as três coletas. Ribeirão Preto, 2007

\begin{tabular}{lrrrrrrrrr}
\hline \multicolumn{10}{c}{ MSSA } \\
\hline \multirow{2}{*}{ Mupirocina } & \multicolumn{1}{c}{$\mathbf{1}^{\mathbf{a}}$} & \multicolumn{2}{c}{$\mathbf{2}^{\mathbf{a}}$} & \multicolumn{2}{c}{$\mathbf{3}^{\mathbf{a}}$} & \multicolumn{2}{c}{ Total } \\
& n. $^{\circ}$ & \multicolumn{1}{c}{$\%$} & n. $^{\circ}$ & $\%$ & n. $^{\circ}$ & $\%$ & n. & \multicolumn{1}{c}{$\%$} \\
\hline Sensível & 48 & 87,3 & 40 & 88,9 & 33 & 97,1 & $\mathbf{1 2 1}$ & $\mathbf{9 0 , 3}$ \\
Resistente & 7 & 12,7 & 5 & 11,1 & 1 & 2,9 & $\mathbf{1 3}$ & $\mathbf{9 , 7}$ \\
\hline Total & $\mathbf{5 5}$ & $\mathbf{1 0 0 , 0}$ & $\mathbf{4 5}$ & $\mathbf{1 0 0 , 0}$ & $\mathbf{3 4}$ & $\mathbf{1 0 0 , 0}$ & $\mathbf{1 3 4}$ & $\mathbf{1 0 0 , 0}$ \\
\hline
\end{tabular}

\section{DISCUSSÃO}

Pesquisa realizada em vários hospitais em Riyadh, Arábia Saudita, indicam que a resistência à mupirocina é maior

Tabela 1 - Staphylococcus aureus isolados em saliva de profissionais de enfermagem ( $\mathrm{n}=158)$, segundo as três coletas e sensibilidade ou resistência à meticilina. Ribeirão Preto, 2007- 8

\begin{tabular}{lccrrrrrrr}
\hline \multirow{2}{*}{ Staphylococcus aureus } & \multicolumn{3}{c}{$\mathbf{1}^{\mathbf{a}}$} & \multicolumn{2}{c}{$\mathbf{2}^{\mathbf{a}}$} & \multicolumn{3}{c}{$\mathbf{3}^{\mathbf{a}}$} & \multicolumn{2}{c}{ Total } \\
& n. ${ }^{\circ}$ & $\%$ & n. $^{\circ}$ & $\%$ & n. & & $\%$ & \multicolumn{1}{c}{.$^{\circ}$} & $\%$ \\
\hline MRSA & 18 & 24,7 & 3 & 6,3 & 3 & 8,1 & $\mathbf{2 4}$ & $\mathbf{1 5 , 2}$ \\
MSSA & 55 & 75,3 & 45 & 93,7 & 34 & 91,9 & $\mathbf{1 3 4}$ & $\mathbf{8 4 , 8}$ \\
\hline Total & $\mathbf{7 3}$ & $\mathbf{1 0 0 , 0}$ & $\mathbf{4 8}$ & $\mathbf{1 0 0 , 0}$ & $\mathbf{3 7}$ & $\mathbf{1 0 0 , 0}$ & $\mathbf{1 5 8}$ & $\mathbf{1 0 0 , 0}$ \\
\hline
\end{tabular}


quando o Staphylococcus aureus é resistente à meticilina ${ }^{(16)}$, fato este confirmado em nosso estudo.

Considerando a mupirocina como o antimicrobiano de escolha para a descolonização de indivíduos com MRSA, estudo revela importante resistência a este fármaco e sua associação com a política de uso ${ }^{(5)}$. Em uma investigação epidemiológica realizada no Brasil, foi observada significativa resistência à mupirocina em Staphylococcus aureus isolados em dois hospitais, um no Rio de Janeiro (RJ) e outro em Uberlândia (MG). Diferentes condutas foram empregadas no uso do referido antimicrobiano. A resistência observada na primeira instituição mencionada foi de $63 \%$ onde a descolonização foi feita com uso tópico extensivo da mupirocina. No outro hospital, o uso foi restrito sendo detectado $6,1 \%$ de resistência ${ }^{(5)}$.

Em outra região distante geograficamente, em Trinidad, (Porto Espanha), um país da América Central, constituído de duas ilhas, localizado no mar do Caribe com sua capital situada em Trinidad, também foi evidenciada a emergente resistência à mupirocina entre cepas de MRSA isolados em hospital geral, após 20 anos de uso contínuo(17). Na Europa, foram observadas altas taxas de resistência à mupirocina em 19 hospitais, tendo os pesquisadores concluído que, para manter a prevalência relativamente baixa, é prudente o uso restrito por meio de medidas estratégicas de controle(18). Em hospitais canadenses, foi observado um aumento na resistência de 1,6\% para 7,0\% em cinco anos de vigilância $(p<0,001)(10)$.

Em diferentes áreas da Malásia, que têm uma política de uso restrito de mupirocina, observou-se baixa porcentagem de resistência $(1,25 \%)$ entre 400 MRSA pesquisados ${ }^{(19)}$.

Comparando a instituiçãoinvestigada com as diferentes localidades apresentadas, podemos constatar que a resistência à mupirocina na instituição pesquisada é relativamente alta $(18,98 \%)$ em relação àquelas que fazem uso restrito de mupirocina. Mas, o hospital pesquisado difere dos demais por não fazer uso da mupirocina para descolonização e apresentar altas taxas de resistência. O hospital em estudo faz rotineiramente o screening e a descolonização, com sulfadiazina de prata a $1 \%$ e, recentemente, iniciou-se a descolonização de todos os pacientes com cultura positiva para MRSA, porém não fazendo o screening, mas, sim, utilizando as culturas solicitadas pelos médicos dos pacientes. No hospital em estudo, o método de descolonização adotado inclui o banho com clorexidina degermante $2 \%$ e a aplicação nasal de sulfadiazina de prata $1 \%$ (cinco dias), já que não dispõe de mupirocina e também pelo receio de resistência a esta droga.

É relevante apontar que o uso da mupirocina faz parte de uma estratégia global de controle do MRSA que inclui também o banho com clorexidina degermante e as precauções de contato para o portador de MRSA, a higienização rigorosa das mãos dos profissionais de saúde, o uso adequado de antibióticos sistêmicos, entre outras medidas. A recomendação mais aplicada em hospitais é a preconizada pelos $\mathrm{CDC}^{(20)}$, que aborda o sistema de precauções e isolamento. Salienta-se, ainda, as orientações específicas para os portadores de bactérias multidrogarresistentes ${ }^{(22)}$ que recomendam não descolonizar rotineiramente o portador de MRSA, mas avaliar circunstâncias específicas nas quais deve ser considerada ${ }^{(21)}$.

Portanto, seu uso deve ser bem avaliado, considerando a necessidade de reflexão sobre o emprego da mupirocina para descolonização, e a probabilidade de desenvolvimento de resistência relacionada à política de uso $^{(5)}$.

Avaliações individualizadas e situações especiais devem ser consideradas entre os profissionais.

Consideramos que primeiro será necessário conhecer o perfil de sensibilidade aos antimicrobianos da instituição, inclusive, da mupirocina, para posteriormente realizar o planejamento das ações de controle dos microorganismos multirresistentes, entre elas, a medida de descolonização do portador de MRSA com mupirocina. Devemos também considerar o uso de outros medicamentos testados e de comprovada eficácia.

A contribuição deste estudo ocorre pela identificação do perfil de sensibilidade a este antimicrobiano testado, mupirocina, que vem mais uma vez nos alertar a respeito da problemática da multirresistência e da necessidade de uma avaliação criteriosa paro uso de antimicrobianos, mesmo que tópicos, na prática diária.

\section{CONCLUSÕES}

Ao avaliarmos a saliva dos profissionais de enfermagem de um hospital de nível terciário, evidenciamos a presença de Staphylococcus aureus sensíveis e o perfil de resistência à meticilina e à oxacilina. Dentre os 356 profissionais testados, obtivemos o seguinte resultado:

- De 1.068 amostras de saliva de profissionais de enfermagem processadas, foi identificado Staphylococcus aureus em 158 (14,8\%).

- Dos 158 isolados, 24 foram caracterizados, como MRSA e 134 como MSSA

- Um total de 30/158 (19\%) apresentou-se resistentes à mupirocina, sendo 17 MRSA e 13 MSSA.

A efetividade das medidas de descolonização na redução e controle das infecções e, consequentemente, na redução da disseminação de MRSA vem sendo demonstrada. A resistência à mupirocina tem sido identificada em Staphylococcus aureus isolados nos serviços de saúde, incluindo o hospital deste estudo e considerando os, benefícios relacionados à descolonização do portador de MRSA é necessário ponderar sobre sua utilização. 
É importante que o uso racional e controlado desse antimicrobiano, aliado à monitorização rotineira da susceptibilidade aos Staphylococcus aureus isolados de pacientes ou profissionais da saúde sejam estratégias adotadas pelas instituições. Medidas de controle para preservar a eficácia desse antimicrobiano a longo prazo devem ser implementadas, dentre estas, o uso ponderado do antimicrobiano para descolonização, em situações especiais, visto que a utilização rotineira não é recomendada, entretanto há de se considerar que, em circunstâncias específicas, ele pode ser recomendado. Também novas pesquisas devem ser realizadas para

\section{REFERÊNCIAS}

1. Klevens RM, Morrison MA, Nadle J, Petit S, Gershman K, Ray S, Harrison LH, Lynfield R, Dumyati G, Townes JM, Craig AS, Zell ER, Fosheim GE, McDougal LK, Carey RB, Fridkin SK; Active Bacterial Core surveillance (ABCs) MRSA Investigators. Invasive methicillin-resistant Staphylococcus aureus infections in the United States. JAMA. 2007:298(15):1763-71. Comment in: JAMA. 2008;299(5):519; author reply 519-20. JAMA. 2007;298(15):1803-4.

2. Elixhauser A, Steiner C. (AHRQ). Infections with MethicillinResistant Staphylococcus aureus (MRSA) in U.S. Hospitals, 1993-2005. HCUP Statistical Brief 35. July 2007. Agency for Healthcare Research and Quality, Rockville, MD. http:// www.hcup-us.ahrq.gov/reports/statbriefs/sb35.pdf

3. Coia JE, Duckworth GJ, Edwards DI, Farrington M, Fry C, Humphreys H, Mallaghan C, Tucker DR; Joint Working Party of the British Society of Antimicrobial Chemotherapy; Hospital Infection Society; Infection Control Nurses Association. Guidelines for the control and prevention of methicillin-resistant Stapbylococcus aureus (MRSA) in healthcare facilities. J Hosp Infect. 2006. 63 Suppl 1:S1-44. Review. Erratum in: J Hosp Infect. 2006;64(1):97-8.

4. Wertheim HF, Vos MC. Can mupirocin prevent methicillinresistant Staphylococcus aureus infections? Crit Care. 2005;9(3):257-8.

5. Netto dos Santos KR, de Souza Fonseca L, Gontijo Filho PP. Emergence of high-level mupirocin resistance in methicillin-resistant Staphylococcus aureus isolated from Brazilian university hospitals. Infect Control Hosp Epidemiol. 1996;17(12):813-6. Comment in: Infect Control Hosp Epidemiol. 1996;17(12):775-9.

6. Robicsek A, Beaumont JL, Paule SM, Hacek DM, Thomson RB Jr, Kaul KL, et al. Universal surveillance for methicillinresistant Staphylococcus aureus in 3 affiliated hospitals. Ann Intern Med. 2008;148(6):409-18.

7. Unemura Y, Ishida Y, Suzuki Y, Yanaga K. Impact of prophylactic mupirocin for radical esophagectomy. J Infect Chemother. 2006;12(5):257-63.

8. Trautmann M, Stecher J, Hemmer W, Luz K, Panknin HT. Intranasal mupirocin prophylaxis in elective surgery. A review of published studies. Chemotherapy. 2008;54(1):9-16.

9. Simor AE, Stuart TL, Louie L, Watt C, Ofner-Agostini M, Gravel D, Mulvey M, Loeb M, McGeer A, Bryce E, Matlow A; Canadian Nosocomial Infection Surveillance Program. Mupirocin-resistant, methicillin-resistant Staphylococcus aureus strains in Canadian hospitals. Antimicrob Agents Chemother. 2007;51(11):3880-6.

10. Sesso R, Barbosa D, Leme IL, Sader H, Canziani ME, Manfredi S, et al. Staphylococcus aureus prophylaxis in identificar e testar outros medicamentos e/ou medidas eficazes de controle da disseminação de MRSA associada às medidas já existentes e comprovadas.

Esta orientação também se faz necessária para proteção ocupacional do trabalhador e para evitar sua disseminação no ambiente hospitalar e prevenir um problema mundial de saúde pública.

Constatamos que a medida de controle de MRSA por meio da descolonização com mupirocina está se tornando uma medida ineficaz, pois ocorre resistência a esse antimicrobiano, portanto, necessitamos testar novos medicamentos e usá-los criteriosamente.

hemodialysis patients using central venous catheter: effect of mupirocin ointment. J Am Soc Nephrol. 1998;9(6):108592.

11. Moreira M, Freitas MR, Martins ST, Castelo A, Medeiros EA. Efficacy of a program of prevention and control for methicillin-resistant Staphylococcus aureus infections in an intensive-care unit. Braz J Infect Dis. 2007;11(1):57-62.

12. Udo EE, Jacob LE, Mathew B. The spread of a mupirocinresistant/methicillin-resistant Staphylococcus aureus clone in Kuwait hospitals. Acta Trop. 2001;80(2):155-61.

13. Watanabe H, Masaki H, Asoh N, Watanabe K, Oishi K, Kobayashi S, et al. Low concentrations of mupirocin in the pharynx following intranasal application may contribute to mupirocin resistance in methicillin-resistant Staphylococcus aureus. J Clin Microbiol. 2001;39(10):3775-7.

14. Udo EE, Jacob LE, Mathew B. Genetic analysis of methicillin-resistant Staphylococcus aureus expressing high- and low-level mupirocin resistance. J Med Microbiol. 2001;50(10):909-15.

15. Clinical and Laboratory Standards Institute / CLSI. Performance standards for antimicrobial susceptibility testing. Fifteenth informational supplement. CLSI document M100-S15. Wayne, PA: CLSI/NCCLS, 2005.

16. Baddour MM, Abuelkheir MM, Fatani AJ. Trends in antibiotic susceptibility patterns and epidemiology of MRSA isolates from several hospitals in Riyadh, Saudi Arabia. Ann Clin Microbiol Antimicrob. 2006;5:30.

17. Orrett FA. The emergence of mupirocin resistance among clinical isolates of methicillin-resistant Staphylococcus aureus in Trinidad: a first report. Jpn J Infect Dis. 2008;61(2):107-10.

18. Schmitz FJ, Lindenlauf E, Hofmann B, Fluit AC, Verhoef J, Heinz HP, Jones ME. The prevalence of low- and highlevel mupirocin resistance in staphylococci from 19 European hospitals. J Antimicrob Chemother. 1998;42(4):489-95.

19. Norazah A, Koh YT, Ghani Kamel A, Alias R, Lim VK. Mupirocin resistance among Malaysian isolates of methicillin-resistant Staphylococcus aureus. Int J Antimicrob Agents. 2001;17(5):411-4.

20. Siegel JD, Rhinehart E, Jackson M, Chiarello L, Healthcare Infection Control Practices Advisory Committee. 2007 guideline for isolation precautions: preventing transmission of infectious agents in healthcare settings. Transmissionbased precautions. Atlanta (GA): Centers for Disease Control and Prevention (CDC); 2007.

21. Siegel JD, Rhinehart E, Jackson M, Chiarello L, Healthcare Infection Control Practices Advisory Committee. Management of multidrug-resistant organisms in healthcare settings. Atlanta (GA): Centers for Disease Control and Prevention; 2006. 